\title{
Slowly rotating black holes in quasitopological gravity
}

\author{
Octavio Fierro, ${ }^{1}$ Nicolás Mora, ${ }^{2}$ and Julio Oliva ${ }^{2}$ \\ ${ }^{1}$ Departamento de Matemática y Física Aplicadas, Universidad Católica de la Santísima Concepción, \\ Alonso de Ribera 2850, Concepción, Chile \\ ${ }^{2}$ Departamento de Física, Universidad de Concepción, Casilla 160-C, Concepción, Chile
}

(Received 11 December 2020; accepted 4 February 2021; published 2 March 2021)

\begin{abstract}
While cubic quasitopological gravity is unique, there is a family of quartic quasitopological gravities in five dimensions. These theories are defined by leading to a first-order equation on spherically symmetric spacetimes, resembling the structure of the equations of Lovelock theories in higher dimensions, and are also ghost free around anti-de Sitter (AdS). Here we construct slowly rotating black holes in these theories and show that the equations for the off-diagonal components of the metric in the cubic theory are automatically of second order, while imposing this as a restriction on the quartic theories allows to partially remove the degeneracy of these theories, leading to a three-parameter family of Lagrangians of order four in the Riemann tensor. This shows that parallel with Lovelock theory observed on spherical symmetry extends to the realm of slowly rotating solutions. In the quartic case, the equations for the slowly rotating black hole are obtained from a consistent, reduced action principle. These functions admit a simple integration in terms of quadratures. Finally, in order to go beyond the slowly rotating regime, we explore the consistency of the KerrSchild Ansatz in cubic quasitopological gravity. Requiring the spacetime to asymptotically match with the rotating black hole in general relativity, for equal oblateness parameters, the Kerr-Schild deformation of an AdS vacuum, does not lead to a solution for generic values of the coupling. This result suggests that in order to have solutions with finite rotation in quasitopological gravity, one must go beyond the Kerr-Schild Ansatz.
\end{abstract}

DOI: 10.1103/PhysRevD.103.064004

\section{INTRODUCTION}

In order to construct a gravitational Lagrangian beyond the Einstein-Hilbert combination, leading to second-order field equations for the metric tensor of a generic spacetime, one is forced to go beyond four dimensions. Lovelock theory emerge as the general, diffeomorphism invariant gravitational action that is constructed with the metric as the unique field and leads to second-order field equations [1]. Each Lovelock term has a simple interpretation as the dimensional continuation of a lower dimensional Euler density [2] and has received considerable attention during the last decades since it permits to study in a controlled framework, the effects of higher curvature terms in the gravitational dynamics (see, e.g., [3-5] and references therein). The Einstein-Gauss-Bonnet Lagrangian, which is the first Lovelock theory beyond general relativity (GR), also appears in the low-energy limit of heterotic string theory [6]. In such setup, the higher curvature terms are consistent only in a perturbative regime [7], an

Published by the American Physical Society under the terms of the Creative Commons Attribution 4.0 International license. Further distribution of this work must maintain attribution to the author(s) and the published article's title, journal citation, and DOI. Funded by SCOAP ${ }^{3}$. interpretation that is supported by studies of the initial value problem in this framework; see, e.g., [8-10]. In this scenario, going beyond quadratic corrections may lead to higher curvature combinations outside of the Lovelock family, even after using the field redefinitions proper of the perturbative approach [11]. Furthermore, the number of independent, nontrivial Lovelock terms is bounded from above by $n<D / 2$, where $n$ is the degree of homogeneity of the Lovelock combination that goes as Riem ${ }^{n}$. In this manner, in a five-dimensional holographic setup and within the realm of Lovelock gravities, one cannot go beyond Riem $^{2}$ terms. Generic higher curvature terms will propagate unhealthy degrees of freedom even around the maximally symmetric vacuum of the theory, and in such setups it is usually impossible to construct analytic, spherically symmetric black holes.

In [12], a new five-dimensional combination of cubic terms was originally introduced, which has interesting properties: (1) it leads to second-order field equations on a generic spherically symmetric spacetime, (2) it possesses a Birkhoff's theorem, namely, spherical symmetry implies staticity for generic values of the couplings, and (3) it possesses asymptotically flat and (anti)-de Sitter (A)dS black holes that are determined by the solution of an algebraic cubic equation, mimicking the structure of cubic Lovelock theories in dimension greater than 6 . The authors 
of [13] arrived to the same cubic combination in dimension five and dubbed it quasitopological gravity (a terminology that hereafter we adopt). They found that even though the field equations are of fourth order for generic backgrounds, the perturbations around an AdS vacuum of the theory are of second order. Actually, the equation for the graviton on AdS in quasitopological gravity reduces to that in GR, with an effective Newton's constant. In the original reference [12], it was actually recognized that the cubic combination was a particular case of a family of Lagrangians containing terms of the form Riem $^{k}$ in dimension $D=2 k-1$, which can be written as

$$
\tilde{\mathcal{L}}_{k}=\frac{1}{2^{k}}\left(\frac{1}{D-2 k+1}\right) \delta_{c_{1} d_{1} \cdots c_{k} d_{k}}^{a_{1} b_{1} \cdots a_{k} b_{k}}\left(C_{a_{1} b_{1}}^{c_{1} d_{1}} \cdots C_{a_{k} b_{k}}^{c_{k} d_{k}}-R_{a_{1} b_{1}}^{c_{1} d_{1}} \cdots R_{a_{k} b_{k}}^{c_{k} d_{k}}\right)-c_{k} C_{a_{1} b_{1}}^{a_{k} b_{k}} C_{a_{2} b_{2}}^{a_{1} b_{1}} \cdots C_{a_{k} b_{k}}^{a_{k-1} b_{k-1}}
$$

Here $C_{a}{ }^{b} c d$ is the Weyl tensor and

$$
c_{k}=\frac{(D-4) !}{(D-2 k+1) !} \frac{[k(k-2) D(D-3)+k(k+1)(D-3)+(D-2 k)(D-2 k-1)]}{\left[(D-3)^{k-1}(D-2)^{k-1}+2^{k-1}-2(3-D)^{k-1}\right]} .
$$

The cubic quasitopological theory is therefore obtained by setting $D=5$ and $k=3$ in Eq. (1) once the generalized Kronecker delta has been expanded. Notice that no singular limit has to be taken in order to make sense of the cubic combination (1) in $D=5$, since after the generalized Kronecker delta is expanded, the would-be singular factor is canceled.

The main objective of the present paper is to fill a gap and to study rotating solutions of quasitopological gravities. In the presence of higher curvature terms, rotating solutions are notoriously difficult to study and often one is forced to rely on approximate and/or numerical schemes. For example, even in the simplest Lovelock theory beyond GR, the Einstein-Gauss-Bonnet theory, for generic values of the couplings, the rotating black hole solutions are only known numerically $[14,15]$ and perturbative in the angular momentum [16,17]. Analytic, rotating solutions can be constructed for the special values of couplings that allow formulating the theory as Chern-Simons theory for the AdS group [18] (see also [19]). The exact solution in five dimensions is noncircular [20] and may have an intricate causal structure.

In five dimensions, one can also construct quartic quasitopological combinations. The first of these combinations was presented in [21]. At the level of spherical symmetry, the cubic theory in five dimensions is unique, while there is a family of quartic theories leading to second-order field equations. A family of quintic quasitopological theories was also constructed in [22]. Surprisingly, in [23], it has been recently realized that a recursive method exists which permits to construct quasitopological theories of arbitrary order.

In this paper, we construct slowly rotating solutions of cubic and quartic quasitopological theories. We show that requiring the equations for the rotating function profile to be of second order, further restricts the couplings of the quartic combinations. We also provide no-go results about the usefulness of the Kerr-Schild Ansatz beyond the slowly rotating regime.

This paper is organized as follows: in Sec. II, we review the main properties of cubic quasitopological gravity in regards to the structure of vacua and static black holes. In Sec. III, in order to approach the problem of the rotating solutions in cubic quasitopological gravity, we construct the slowly rotating black holes with two angular momenta. The functions controlling the rotation fulfill a second-order system that can be integrated in terms of quadratures. Section IV contains an extension of these results for quartic quasitopological theories in five dimensions, and remarkably we show that requiring a second-order equation for the off-diagonal terms allows to further constrain the coefficients of the quartic terms, leading to four different families of theories. As a first attempt to go beyond the slowly rotating regime, we explore the Kerr-Schild Ansatz in Sec. V. For the cubic case, we show that it is impossible to accommodate a rotating solution on this Ansatz for generic values of the couplings, requiring the leading behavior at infinity to be that of GR, as it occurs on spherical symmetry. After proving this no-go result in Sec. VII, we conclude and provide further comments.

\section{REVIEWING THE VACUA AND STATIC BLACK HOLES IN CUBIC QUASITOPOLOGICAL GRAVITY}

We will work with the theory

$$
\begin{aligned}
I_{C Q T G}= & \int \sqrt{-g} d^{5} x\left(R-2 \Lambda_{0}+\alpha_{2}\left(R^{2}-4 R_{a b} R^{a b}+R_{a b c d} R^{a b c d}\right)+\alpha_{3}\left(R_{a b c d} R^{b e d f} R_{e}{ }^{a} f^{c}-\frac{9}{7} R_{a b c d} R^{a b c e} R_{e}^{d}+\frac{3}{8} R_{a b c d} R^{a b c d} R\right.\right. \\
& \left.\left.+\frac{15}{7} R_{a b c d} R^{a c} R^{b d}+\frac{18}{7} R_{a}^{b} R_{b}^{c} R_{c}^{a}-\frac{33}{14} R_{a b} R^{a b} R+\frac{15}{56} R^{3}\right)\right) .
\end{aligned}
$$



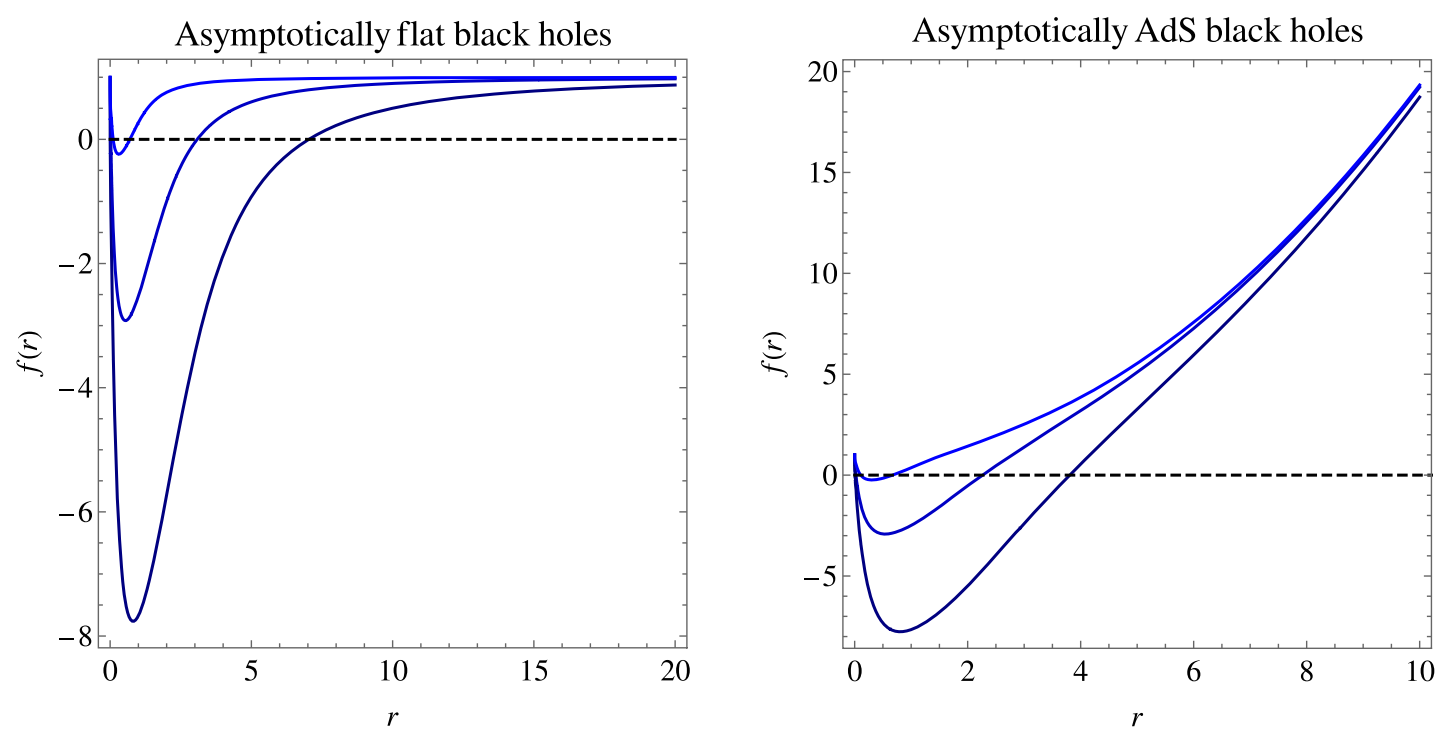

FIG. 1. The figure depicts the function $f(r)$ for asymptotically flat black holes (left panel) and asymptotically AdS black holes (right panel) for different values of the mass parameter $m$ in Eq. (5), in particular $m=1,10,50$. The profiles were obtained for small values of the higher curvature couplings $\alpha_{2}=0.25$ and $\alpha_{3}=-0.01$, while for the asymptotically AdS black holes the bared cosmological term $\Lambda_{0}$ was set equal to -1 .

The metric

$$
d s^{2}=-f(t, r) d t^{2}+\frac{d r^{2}}{g(t, r)}+r^{2} d \Sigma_{3}^{2}
$$

is the most general Ansatz for a spacetime that is compatible with the possible isometries of the three-dimensional Euclidean manifold $\Sigma_{3}$, which we assume of constant curvature $\gamma= \pm 1,0$. As shown in [12], the field equations of quasitopological gravity imply, up to a redefinition of the coordinate $t$, that is, $g(t, r)=f(t, r)=f(r)$ where the function $f(r)$ is a solution of the following cubic polynomial equation:

$$
\begin{aligned}
& 24 \alpha_{3}(f-\gamma)^{3}+84 \alpha_{2} r^{2}(f-\gamma)^{2}-42 r^{4}(f-\gamma) \\
& \quad-7 r^{6} \Lambda_{0}=42 m r^{2},
\end{aligned}
$$

where $m$ is an integration constant that will determine the energy content of the spacetime. These black holes can also be dressed by a conformal scalar hair [24,25]. The curvature radii $l$ of the maximally symmetric (A)dS solutions of the theory are determined from (5) setting $m=0$ and $f=\gamma-\Lambda_{\mathrm{eff}} r^{2} / 6$, leading to

$\Upsilon\left[\Lambda_{\text {eff }}\right]:=\alpha_{3} \Lambda_{\text {eff }}^{3}-21 \alpha_{2} \Lambda_{\text {eff }}^{2}-63 \Lambda_{\text {eff }}+63 \Lambda_{0}=0$.

Unless otherwise stated, we will assume that the couplings of the theory are generic, which implies that there is always a solution of (6) that goes to $\Lambda_{\text {eff }}^{(i)} \rightarrow \Lambda_{0}$, as the couplings of the quadratic and cubic terms in the action vanish. ${ }^{1}$ Here $i \in\{1,2,3\}$. The three possible solutions of (5) approach each of the three possible asymptotic curvatures $\Lambda_{\text {eff }}^{(i)}$. Namely, the three solutions of (5) behave asymptotically as

$$
f^{(i)}(r)=-\frac{\Lambda_{\mathrm{eff}}^{(i)}}{6} r^{2}+\gamma-\frac{m}{r^{2}}+O\left(r^{-3}\right),
$$

and as usual we define the GR branch as the one that smoothly connects with the Einstein solution as

$$
\lim _{\left(\alpha_{3}, \alpha\right) \rightarrow 0} \Lambda_{\mathrm{eff}}^{(i)}\left(\kappa, \alpha, \Lambda_{0}\right) \rightarrow \Lambda_{0}
$$

Hereafter, we will focus on this branch.

Even though the solutions of the cubic equation can be written explicitly in terms of radicals, they are not particularly illuminating and therefore we only present some examples in Fig. 1.

As observed in [12], the polynomial (5) resembles the structure of Wheeler's polynomial in cubic Lovelock theory [27,28], formally replacing $D=5$ after a $(D-5)$ factor is absorbed in the coupling of the cubic Lovelock terms (see

\footnotetext{
${ }^{1}$ Two of these vacua coincide provided the couplings fulfill the constraint $\alpha_{3}^{2} \Lambda_{0}^{2}-14\left(\alpha_{2} \Lambda+\frac{1}{3}\right) \alpha_{3}-\frac{49}{9} \alpha_{2}^{2}\left(4 \alpha_{2} \Lambda_{0}+3\right)=0$, while the three of them degenerate provided $\alpha_{2} \Lambda_{0}=-1$ and $\alpha_{3}=-\frac{7}{3 \Lambda_{0}}$, leading to a single cosmological constant $\Lambda=3 \Lambda_{0}$. These cases are incompatible with the interpretation of the higher curvature terms as perturbative corrections to GR, but may allow to enlarge the space of solutions or even enlarge the symmetry group of the theory as it happens in Lovelock theories [26].
} 
also Eq. (5.8) of [29] as well as [30-32]). The same pattern extends to quasitopological gravities of higher degree $([22,23])$.

In the next section, we move forward and construct the slowly rotating black hole solutions of cubic quasitopological gravity.

\section{SLOWLY ROTATING BLACK HOLES IN QUASITOPOLOGICAL GRAVITY}

The metric Ansatz that accommodates the slowly rotating black hole solution of quasitopological gravity is

$$
\begin{aligned}
d s^{2}= & -f(r) d t^{2}+\frac{d r^{2}}{f(r)}+r^{2}\left(\frac{d \mu^{2}}{1-\mu^{2}}+\mu d \phi^{2}+(1-\mu)^{2} d \psi^{2}\right) \\
& -a_{1} \mu^{2} r^{2} g_{1}(t, r) d t d \phi-a_{2}\left(1-\mu^{2}\right) r^{2} g_{2}(t, r) d t d \psi,
\end{aligned}
$$

with $\quad-\infty<t<\infty, \quad-1 \leq \mu \leq 1, \quad$ and $\quad 0 \leq \phi \leq 2 \pi$, $0 \leq \psi \leq 2 \pi$. Here $a_{1}$ and $a_{2}$ are the rotation parameters which we will consider small. Notice that we have chosen the static metric to be spherically symmetric, even though the present analysis can be extended for arbitrary curvature of the horizon of the static black hole, which may lead to rotating, topological black branes as in GR in four dimensions [33]. As usual, expanding the field equations in powers of $a_{1}$ and $a_{2}$ leads, to the lowest order, to the same equation for $f(r)$ that implies (5), and we write it here for future purposes in its differential form

$$
\begin{aligned}
& \left(\frac{12(f-1)^{2} \alpha_{3}}{7 r^{2}}+4(f-1) \alpha_{2}-r^{2}\right) \frac{d f}{d r} \\
& -\frac{8}{7}(f-1)^{3} \frac{\alpha_{3}}{r^{3}}-\frac{2}{3} r^{3} \Lambda_{0}-2 r(f-1)=0 .
\end{aligned}
$$

It is simpler to work with the function $\varphi(r)$ such that

$$
\varphi(r)=\frac{1-f(r)}{r^{2}} .
$$

At linear order in the rotation parameters, one obtains the equations for $g_{1}(t, r)$ and $g_{2}(t, r)$ that allow to construct the slowly rotating black hole solutions. The equation is

$$
A(\varphi, r) \frac{d^{2} g_{i}}{d r^{2}}+B(\varphi, r) \frac{d g_{i}}{d r}=0
$$

and reduces manifestly to a second-order system. Here

$$
A(\varphi, r)=-\frac{\left(1-r^{2} \varphi\right)}{42}\left(30 \kappa\left(r^{2} \varphi^{\prime}\right)^{\prime}+r^{2}(12 \kappa \varphi-28 \alpha \varphi-7)\right),
$$

$$
\begin{aligned}
B(\varphi, r)= & A^{\prime}(\varphi, r)-\frac{\left(r^{3} \varphi^{\prime 2} \varphi+3\right)}{42 r}\left(30 \kappa\left(r^{2} \varphi^{\prime}\right)^{\prime}\right. \\
& \left.+r^{2}(12 \kappa \varphi-28 \alpha \varphi-7)\right) .
\end{aligned}
$$

This simplification is remarkable since a generic perturbation of the static black hole metric may lead to a fourthorder system [13]; nevertheless, the perturbation that allows constructing the slowly rotating solutions is determined by a second-order system, as in general relativity and Lovelock theories.

It is evident that there is a simple solution of the system (12) given by $g_{1}=g_{1}(t)$ and $g_{2}=g_{2}(t)$, both functions being arbitrary. It is simple to show that at linear order in the rotation parameters these solutions are pure gauge since one can define

$$
\phi=\tilde{\phi}-\frac{a_{1}}{2} \int g_{1}(t) d t \text { and } \psi=\tilde{\psi}-\frac{a_{2}}{2} \int g_{1}(t) d t,
$$

such that the metric (9) reduces to a static metric up to terms which are quadratic on the rotation parameters. This change of coordinates is obstructed when the functions $g_{1}$ and $g_{2}$ depend on the radial coordinate. We therefore solve Eq. (12) by setting $g_{1}=g_{1}(r)$ and $g_{2}=g_{2}(r)$. The equations for the $g_{i}(r)$ can be integrated in terms of quadratures, since they imply the remarkably compact expression

$\frac{g_{i}^{\prime \prime}(r)}{g_{i}^{\prime}(r)}=-\left(\ln \left[30 \alpha_{3} r^{3}\left(r^{2} \varphi^{\prime}\right)^{\prime}-\frac{28 m}{\varphi^{\prime}}\right]\right)^{\prime} \quad$ with $\quad i=1,2$,

therefore

$$
g_{i}(r)=B_{i} \int \frac{\varphi^{\prime} d r}{30 \alpha_{3} r^{3} \varphi^{\prime}\left(r^{2} \varphi^{\prime}\right)^{\prime}-28 m}+C_{i},
$$

where $B_{i}$ and $C_{i}$ are integration constants. It is important to notice that in the absence of the cubic quasitopological term, the expression for $g_{i}(r)$ can be integrated in a closed form. This was done in $[16,17]$. It is interesting to notice also that the function $g_{i}(r)$ depends on the Gauss-Bonnet coupling only through $\varphi$, a feature that is shared by generic Lovelock theories (see Appendix A of [34]). We have depicted some profiles for $f(r)$ and the off-diagonal functions $g(r)$ in Fig. 2.

\section{SLOWLY ROTATING SOLUTIONS FROM SYMMETRIC CRITICALITY AND QUARTIC QUASITOPOLOGICAL GRAVITIES}

It is well known that the field equations obtained from a reduced Lagrangian, obtained evaluating $\mathcal{L}=\sqrt{-g} L$ on a particular Ansatz, are not always the correct field equations $[35,36]$. In a spherically symmetric Ansatz, this principle of symmetric criticality (also known as reduced action or minisuperspace approach), does work correctly in generic diffeomorphism invariant theories, provided one considers two blackening factors. As pointed out in [36], even in spherical symmetry but without assuming staticity, in order 

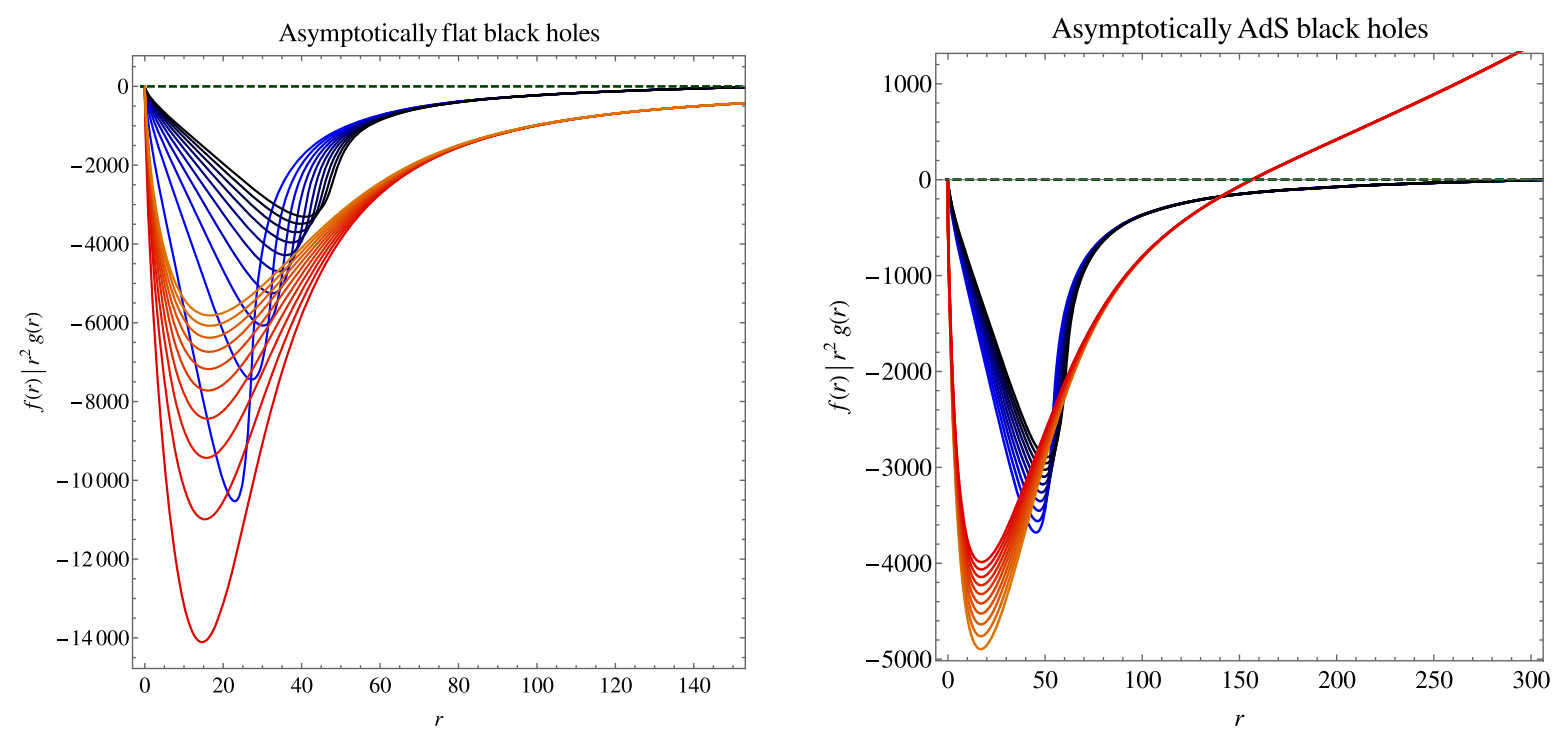

FIG. 2. The figure depicts the profile for $f(r)$ in orange and $g(r)$ in blue for different values of the couplings. We have set the mass parameter as $m=10^{7}$ and $\alpha_{2}=\frac{(14+i)}{100}$ while $\alpha_{3}=-\frac{\alpha_{2}}{25}$, with $i=1, \ldots, 10$. For asymptotically AdS black holes, the bared cosmological term $\Lambda_{0}=-0.1$.

to obtain a consistent reduced action, one must include a function $h(t, r)$, which is pure gauge,

$d s^{2}=-f(t, r) d t^{2}+\frac{d r^{2}}{g(t, r)}+h(t, r) d t d r+r^{2} d \Omega^{2}$.

The Euler-Lagrange equation associated to $h(t, r)$ in the Lovelock and cubic, quartic, and quintic quasitopological gravities are responsible for the staticity of the solution. Inspired by these facts, one can show that the four- and fivedimensional Ansätze,

$$
\begin{aligned}
d s_{4 D}^{2}= & -N^{2}(r) f(r) d t^{2}+\frac{d r^{2}}{f(r)}+r^{2}\left(d \theta^{2}+\sin ^{2} \theta d \phi^{2}\right) \\
+ & h_{1}(r, \theta) d t d \phi \\
d s_{5 D}^{2}= & -N^{2}(r) f(r) d t^{2}+\frac{d r^{2}}{f(r)} \\
& +r^{2}\left(d \theta^{2}+\sin ^{2} \theta d \phi^{2}+\cos ^{2} \theta d \psi^{2}\right) \\
& +g_{1}(r, \theta) d t d \phi+g_{2}(r, \theta) d t d \psi
\end{aligned}
$$

do indeed lead to the correct field equations in the slowly rotating case for general relativity, quadratic gravity, as well as for a generic cubic combination (even beyond the quasitopological Lagrangians). Notice that it is important not to fix the $\theta$ dependence a priori on $g_{i}$. With this evidence at hand, as a matter of fact we apply the principle of symmetric criticality for the quartic quasitopological theories in the slowly rotating regime along the Ansatz (20). It is known that on spherically symmetric spacetimes there is a degeneracy on quartic quasitopological theories, which as shown in the next section is partially removed by requiring second-order field equations for the slowly rotating Ansatz, leading to a quartic quasitopological theory with special properties, even beyond spherical symmetry and considering fluctuations around the spherically symmetric black hole solutions.

\section{QUARTIC QUASITOPOLOGICAL THEORY}

In general, for dimension $d \geq 8$, there are 26 independent scalar terms of the form Riem ${ }^{4}$. Due to multiple curvature identities that appear as one lowers the dimension, the number of independent Riem ${ }^{4}$ combinations is reduced [37], and in five dimensions such list reduces to 20 independent combinations. In order to be able to establish relations in the future in a simple manner between quartic combinations with special properties in different dimensions, even though we will focus on the five-dimensional case, we will work with the complete, redundant, list of 26 scalars. The specific basis of quartic curvature terms $L_{I}$ is given in Appendix A. The action containing up to the quartic quasitopological theory has the form

$$
\begin{aligned}
I & =I_{C Q T G}+I_{Q Q T G}^{(4)} \\
& =I_{C Q T G}+\int \sqrt{-g} d^{5} x \sum_{I=1}^{26} d_{I} L_{I},
\end{aligned}
$$

where we have defined $I_{C Q T G}$ in Eq. (3).

The quartic quasitopological theories are defined such that the field equations on spherical symmetry reduce to a first-order equation for the function $f(r)$, as it occurs in Lovelock theories. This imposes 11 independent 
constraints on the relative couplings $d_{I}$ of the quartic terms. One can further reduce the number of independent couplings by requiring the absence of ghosts when the theory is linearized around a maximally symmetric AdS vacuum. Following the linearization procedure developed in Ref. [38], one can see that for generic higher curvature combinations, the linearized equations around $\mathrm{AdS}$ contain two higher derivative contributions of the form

$$
(2 a+c) \bar{\square} G_{\mu \nu}^{L}+(a+2 b+c)\left(\bar{g}_{\mu \nu} \bar{\square}-\bar{\nabla}_{\mu} \bar{\nabla}_{\nu}\right) R^{L}+\cdots=0,
$$

where the parameters $a, b$, and $c$ depend on the specific Lagrangian (see [38]) and (...) stands for second-order terms. Requiring the absence of ghosts implies two further independent linear restrictions on the couplings. In summary, the 26 couplings $d_{(i)}$ are restricted by 13 independent constraints, leading to 13 remaining couplings. Notice that, as mentioned above, some redundancy coming from algebraic identities of the Riemann tensor has not been removed. Remarkably, after imposing all these constraints, one still has a nontrivial theory.

Now we provide a new argument that allows to further restrict these couplings. Using the reduced action principle described above by the metric Ansatz (20), on the theory constrained by the 13 conditions coming from spherically symmetry and the absence of ghosts around AdS, one obtains an equation for the off-diagonal metric components $g_{1}=\mu^{2} h_{1}(r)$ and $g_{2}=\left(1-\mu^{2}\right) h_{2}(r)$ of the form

$$
\xi_{1} \frac{d^{4} h_{i}}{d r^{4}}+\xi_{2} \frac{d^{3} h_{i}}{d r^{3}}+\xi_{3} \frac{d^{2} h_{i}}{d r^{2}}+\xi_{4} \frac{d h_{i}}{d r}=0, \quad i=1,2
$$

where the $\xi_{j}$ are functions of $r, f(r)$, and the couplings. Requiring the absence of the fourth- and third-order derivatives implies a single new constraint on the couplings, leading to a nontrivial theory with special properties even beyond spherical symmetry.

In summary, requiring the existence of simple spherically symmetric black holes with a single metric function governed by a first-order equation, plus the absence of ghosts around AdS, in addition to the existence of slowly rotating black holes with an off-diagonal metric function determined by a second-order equation, leads to 14 relations between the couplings (see Appendix B).

When the rotation is turned off, as shown in [21], the equation for the blackening factor of the spherically symmetric black holes turns out to be restricted by the following polynomial equation:

$$
\begin{aligned}
& 21 \bar{\alpha}_{4}(f-1)^{4}-24 \alpha_{3}(f-1)^{3} r^{2}-84 \alpha_{2} r^{4}(f-1)^{2} \\
& \quad+42(f-1) r^{6}+7 \Lambda_{0} r^{8}=14 m r^{4},
\end{aligned}
$$

where $\bar{\alpha}_{4}$ is a combination of the quartic invariants given below in Eq. (31). As before, for generic values of the couplings of the terms with different order in the curvature, the quartic theory admits four different vacua with four different values of the effective cosmological constant $\Lambda_{\text {eff }}^{(i)}$ with $i=1,2,3,4$. The four solutions of (24) will asymptotically behave as

$$
f_{(i)}(r)=-\frac{\Lambda_{\mathrm{eff}}^{(i)}}{6} r^{2}+1-\frac{m}{r^{2}}+\cdots
$$

Equation (24) comes from the integration of the following differential equation:

$\frac{\delta \mathcal{L}}{\delta N}=\left(-\frac{2 \bar{\alpha}_{4}(f-1)^{3}}{r^{4}}+\frac{12(f-1)^{2} \alpha_{3}}{7 r^{2}}+4(f-1) \alpha_{2}-r^{2}\right) f^{\prime}+\frac{2 \bar{\alpha}_{4}(f-1)^{4}}{r^{5}}-\frac{8 \alpha_{3}(f-1)^{3}}{7 r^{3}}-2 r(f-1)-\frac{2}{3} \Lambda_{0} r^{3}$,

which as indicated comes from the variation of the reduced action principle, with respect to the function $N$ in the metric (20).

Remarkably, in the context of the quartic quasitopological theories, one can further relate the couplings such that the equation for the off-diagonal components of the metric $g_{(i)}(r)$ takes a very simple, second-order form, leading to

$$
\begin{aligned}
\frac{g_{i}^{\prime \prime}}{g_{i}^{\prime}} & =-\left(\frac{3}{r}+\frac{\left(7 r^{2}\left(r^{2} \beta_{1} \varphi^{\prime 2}+r \beta_{2} \varphi^{\prime} \varphi^{\prime \prime}+\beta_{3} \varphi^{\prime 2}\right)+\left(70 \bar{\alpha}_{4} \varphi+60 \alpha_{3}\right)\left(r^{2} \varphi^{\prime}\right)^{\prime}-\frac{28 m}{r^{3} \varphi^{\prime}}\right)^{\prime}}{7 r^{2}\left(r^{2} \beta_{1} \varphi^{\prime \prime 2}+r \beta_{2} \varphi^{\prime} \varphi^{\prime \prime}+\beta_{3} \varphi^{\prime 2}\right)+\left(70 \bar{\alpha}_{4} \varphi+60 \alpha_{3}\right)\left(r^{2} \varphi^{\prime}\right)^{\prime}-\frac{28 m}{r^{3} \varphi^{\prime}}}\right) \\
& =-\left(\ln \left[7 r^{5}\left(r^{2} \beta_{1} \varphi^{\prime \prime 2}+r \beta_{2} \varphi^{\prime} \varphi^{\prime \prime}+\beta_{3} \varphi^{\prime 2}\right)+r^{3}\left(70 \bar{\alpha}_{4} \varphi+60 \alpha_{3}\right)\left(r^{2} \varphi^{\prime}\right)^{\prime}-\frac{28 m}{\varphi^{\prime}}\right]\right)^{\prime}, \\
g_{i} & =B_{i} \int \frac{\varphi^{\prime} d r}{7 r^{5} \varphi^{\prime}\left(\beta_{1} r^{2} \varphi^{\prime \prime 2}+r \beta_{2} \varphi^{\prime} \varphi^{\prime \prime}+\beta_{3} \varphi^{\prime 2}\right)+r^{3} \varphi^{\prime}\left(70 \bar{\alpha}_{4} \varphi+60 \alpha_{3}\right)\left(r^{2} \varphi^{\prime}\right)^{\prime}-28 m}+C_{i},
\end{aligned}
$$

where 


$$
\begin{aligned}
\bar{\alpha}_{4}=\alpha_{4}\left(d_{13}+2 d_{16}\right. & \left.+4 d_{17}+8 d_{18}+48 d_{19}+d_{20}+4 d_{21}+8 d_{22}+6 d_{23}+22 d_{24}+44 d_{25}+128 d_{26}\right), \\
\beta_{1}= & \alpha_{4}\left(14 d_{20}-30 d_{21}+168 d_{22}+30 d_{23}+303 d_{24}-90 d_{25}-3504 d_{26}\right. \\
& \left.+18 d_{17}+98 d_{18}+216 d_{19}+30 d_{13}+44 d_{16}-16 d_{4}\right), \\
\beta_{2}= & \alpha_{4}\left(156 d_{13}+248 d_{16}+128 d_{17}+604 d_{18}+1632 d_{19}+83 d_{20}-88 d_{21}\right. \\
& \left.+956 d_{22}+208 d_{23}+1828 d_{24}+48 d_{25}-16960 d_{26}-64 d_{4}\right), \\
\beta_{3}= & \alpha_{4}\left(231 d_{13}+398 d_{16}+268 d_{17}+1034 d_{18}+3232 d_{19}+138 d_{20}-38 d_{21}\right. \\
+ & \left.1566 d_{22}+428 d_{23}+3123 d_{24}+908 d_{25}-23040 d_{26}-64 d_{4}\right) .
\end{aligned}
$$

Equation (29) is remarkably simple. The quartic contributions to the denominator of the integrand are of four types. First, there is an $\bar{\alpha}_{4}$ contribution, which adds a term to the previous cubic quasitopological contribution. Notice that $\bar{\alpha}_{4}$ controls the contribution of quartic quasitopological to the spherically symmetric solution (24). It is tempting to conjecture a pattern here for quasitopological gravities of higher order. On top of this, the quartic quasitopological family add three new, independent terms of different kind to the denominator of the integrand of (29). We have checked the linear independence of these combinations after using the 14 constraints mentioned above. One may set zero each of the $\beta s$, further restricting the couplings in favor of the simplicity of the slowly rotating solution. This would lead to the three-parameter family of theories mentioned in the Abstract.

In the next sections, we move beyond the slowly rotating approximations for the cubic quasitopological theories, within the Kerr-Schild Ansatz.

\section{BEYOND SLOWLY ROTATING SOLUTIONS: KERR-SCHILD ANSATZ}

The Kerr-Schild (KS) Ansatz has been a very fruitful arena for the construction of rotating black holes in general relativity in arbitrary dimensions. The whole family of rotating black holes with horizons of hyperspherical topology can be cast in this form [39-44], both in the asymptotically flat case and in the presence of a cosmological constant. The Kerr-Schild Ansatz is defined by

$$
g_{a b}=\tilde{g}_{a b}+F(x) k_{a} k_{b},
$$

where the metric $\tilde{g}_{a b}$ is a seed metric, while the vector field $k$ defines a null and geodesic congruence on the seed spacetime. The latter properties lead to an Einstein tensor that is linear in the function $F$ and its derivatives, facilitating the integration of the field equations. Depending on the expansion, twist, and shear of the congruence, the KS Ansatz can accommodate rotating or static black holes as well as pp waves or (A)dS waves.
This Ansatz has even allowed to construct an intrinsically rotating solution in five dimensions in the presence of a term that is quadratic in the curvature in the context of the Einstein-Gauss-Bonnet theory with a single (A)dS solution [18] and has been explored in some generality in Lovelock gravities in [45]. ${ }^{2}$ Since the Kerr-Schild Ansatz has been useful to construct rotating solutions in higher curvature theories, here we explore this metric Ansatz in the context of the cubic quasitopological gravity.

The Kerr-Schild Ansatz leading to rotating solutions in general relativity in five dimensions has a seed

$$
\begin{aligned}
d \tilde{s}^{2}= & -\frac{\left(1+\frac{r^{2}}{\ell^{2}}\right) \Delta_{\theta} d t^{2}}{\Xi_{a} \Xi_{b}}+\frac{r^{2} \rho^{2} d r^{2}}{\left(1+\frac{r^{2}}{\ell^{2}}\right)\left(r^{2}+a^{2}\right)\left(r^{2}+b^{2}\right)}+\frac{\rho^{2} d \theta^{2}}{\Delta_{\theta}} \\
& +\frac{r^{2}+a^{2}}{\Xi_{a}} \sin ^{2} \theta d \phi^{2}+\frac{r^{2}+b^{2}}{\Xi_{b}} \cos ^{2} \theta d \psi^{2}
\end{aligned}
$$

with $\quad \Xi_{a}=1-\frac{a^{2}}{\ell^{2}}, \quad \Xi_{b}=1-\frac{b^{2}}{\ell^{2}}, \quad \rho^{2}=r^{2}+a^{2} \cos ^{2} \theta+$ $b^{2} \sin ^{2} \theta, \Delta_{\theta}=\Xi_{a} \cos ^{2} \theta+\Xi_{b} \sin ^{2} \theta$, and null and geodesic vector

$$
\begin{aligned}
k_{a} d x^{a}= & \frac{\Delta_{\theta} d t}{\Xi_{a} \Xi_{b}}+\frac{r^{2} \rho^{2} d r}{\left(1+\frac{r^{2}}{\ell^{2}}\right)\left(r^{2}+a^{2}\right)\left(r^{2}+b^{2}\right)} \\
& -\frac{a \sin ^{2} \theta d \phi}{\Xi_{a}}-\frac{a \cos ^{2} \theta d \psi}{\Xi_{b}} .
\end{aligned}
$$

Here the seed metric is that of an AdS spacetime of curvature radius $\ell$, while $a$ and $b$ are the rotation parameters. Since we will be interested in rotating solutions for arbitrary values of the couplings, the leading asymptotic behavior of the metric in quasitopological gravity should be that of GR, with one of the effective cosmological constants. In GR, the function $F(r, \theta)$ is given by

\footnotetext{
${ }^{2}$ See also [46] for the constructions of black holes using the KS Ansatz in DHOST theories, as well as [47] for the construction of slowly rotating black holes in Horndeski theories.
} 
$F(r, \theta)=F_{\mathrm{GR}}(r, \theta)=\frac{2 M}{r^{2}+a^{2} \cos ^{2} \theta+b^{2} \sin ^{2} \theta}$.

For simplicity, hereafter, we focus on the case with $a=b$. This choice enlarges the isometry group of the solution from $\mathbb{R}_{t} \times U(1)^{2}$ to $\mathbb{R}_{t} \times S U(2)$. In this case, asymptotically one has

$$
F_{\mathrm{GR}}(r, \theta)=F_{\mathrm{GR}}(r)=\frac{2 M}{r^{2}}-\frac{M a^{2}}{r^{4}}+O\left(r^{-6}\right) .
$$

As it occurs for spherically symmetric black holes, one requires the asymptotic behavior of the function $F(r)$ to match that of GR with an effective cosmological constant, implying that in the presence of generic higher curvature terms, one must assume an expansion of the form

$$
F(r)=r^{-2}\left(A_{2}+\frac{A_{3}}{r}+\frac{A_{4}}{r^{2}}+\frac{A_{5}}{r^{3}}+O\left(r^{-5}\right)\right)
$$

at infinity.

In cubic quasitopological gravity, the trace of the field equations is a second-order constraint on the metric, for arbitrary spacetimes. In the case of the Kerr-Schild Ansatz (34), with $a=b$ and therefore assuming $F=F(r)$, such constraint reduces to

$$
\begin{aligned}
0= & -30 l^{2}\left(r^{2}+a^{2}\right)^{2}\left[\frac{l^{4} a^{2}}{30} \alpha_{3}\left(a^{2}-5 r^{2}\right)\left(a^{2}+r^{2}\right)^{2} F^{2}+\frac{2 l^{4} a^{2} r \alpha_{3}}{3}\left(r^{4}-1\right) F F^{\prime}\right. \\
& +r^{2}\left(\frac{8 \alpha_{3} l^{4}}{15}\left(a^{2}-\frac{3}{4} r^{2}\right)\left(a^{2}-r^{2}\right) F^{2}-\frac{4 l^{2}}{3}\left(l^{2} \alpha_{2}-\frac{\alpha_{3}}{5}\right)\left(a^{2}+r^{2}\right)^{2}\left(a^{2}-3 r^{2}\right) F\right. \\
& \left.+\left(a^{2}+r^{2}\right)^{4}\left(l^{4}-4 l^{2} \alpha_{2}+\frac{2}{5} \alpha_{3}\right)\right] F^{\prime \prime}+14 \alpha_{3}\left(a^{2}-\frac{5 r^{2}}{7}\right) r l^{6}\left(a^{2}+r^{2}\right)^{3} a^{2} F^{\prime 3} \\
& +40 l^{4}\left(a^{2}+r^{2}\right)^{2}\left[\frac{\alpha_{3} l^{2}}{4}\left(a^{6}-\frac{46}{5} a^{4} r^{2}+\frac{33}{5} a^{2} r^{4}-\frac{12}{5} r^{6}\right) F+r^{2} l^{2}\left(\alpha_{2}-\frac{\alpha_{3}}{5 l^{2}}\right)\left(a^{2}+r^{2}\right)^{2}\left(a^{2}-3 r^{2}\right)\right] F^{\prime 2} \\
& -60 r l^{2}\left(a^{2}+r^{2}\right)^{5}\left[\left(a^{2}+3 r^{2}\right)\left(l^{4}-4 l^{2} \alpha_{2}+\frac{2 \alpha_{3}}{5}\right)+\frac{8 \alpha_{3} l^{4}\left(a^{2}-3 r^{2}\right)}{15\left(a^{2}+r^{2}\right)^{4}}\left(a^{4}-\frac{5}{2} a^{2} r^{2}+\frac{r^{4}}{4}\right) F^{2}\right. \\
& \left.-\frac{4 l^{2}\left(l^{2} \alpha_{2}-\frac{\alpha_{3}}{5}\right)}{3\left(a^{2}+r^{2}\right)^{2}}\left(a^{4}-10 a^{2} r^{2}-3 r^{4}\right) F\right] F^{\prime}+20 r^{2}\left(a^{2}+r^{2}\right)^{6}\left[\left(5 l^{6} \Lambda_{0}+30 l^{4}-60 l^{2} \alpha_{2}+4 \alpha_{3}\right)\right. \\
& \left.-\frac{2 \alpha_{3} r^{2} l^{6}\left(25 a^{4}-22 a^{2} r^{2}+r^{4}\right)}{5\left(a^{2}+r^{2}\right)^{6}} F^{3}-\frac{24 l^{4}\left(5 l^{2} \alpha_{2}-\alpha_{3}\right)\left(3 a^{2}-r^{2}\right) a^{2}}{15\left(a^{2}+r^{2}\right)^{4}} F^{2}-\frac{9 l^{2}\left(5 l^{4}-20 l^{2} \alpha_{2}+2 \alpha_{3}\right)}{5\left(a^{2}+r^{2}\right)} F\right] .
\end{aligned}
$$

The equation $E_{\theta}^{\theta}=0$ coming from the action (21) is of fourth order, and it is useful in the following analysis, but since it is not very illuminating in its complete form we do not provide it here. With these two equations at hand, we can move forward. Assuming an expansion of the form (39) in the $E_{\theta}^{\theta}=0$ equation, the leading and subleading orders in the $r \rightarrow \infty$ expansion lead to

$$
\Upsilon[\Lambda]=\Lambda^{3} \alpha_{3}+90 \Lambda^{2} \alpha_{2}+270 \Lambda-270 \Lambda_{0}=0,
$$

$\frac{d \Upsilon[\Lambda]}{d \Lambda} A_{3}=0$.

Requiring the genericity of the couplings implies $\frac{d \Upsilon[\Lambda]}{d \Lambda} \neq 0$, namely, the curvature radius of the seed is a simple zero of the polynomial $\Upsilon[\Lambda]$. Consequently, $A_{3}=0$. Considering this information in the trace (40) further leads to $A_{5}=A_{7}=0$ as well as $A_{2 n+1}=0$. One also obtains as a consequence of both equations that

$$
\Upsilon^{\prime}[\Lambda]\left(a^{2} A_{2}+A_{4}\right)=0 .
$$

Here we must emphasize that if one imposes relations between the couplings such that $\Upsilon^{\prime}[\Lambda]=0$, new branches of solutions may appear. Actually, from what is known from the spherically symmetric black holes in Lovelock theories with a unique vacuum, it would be natural to expect an asymptotic behavior of the form $r^{-\alpha}$ with $\alpha$ a noninteger number [48].

To the next order in the $r \rightarrow \infty$ expansion, one gets

$$
\Upsilon^{\prime}[\Lambda] A_{6}=\left(a^{4} \Upsilon^{\prime}[\Lambda]-3 \Upsilon^{\prime \prime}[\Lambda]\right) A_{2}
$$

Finally, the following subleading order in the expansion as $r \rightarrow \infty$, leads to an inconsistency, since it implies

$\Upsilon^{\prime}[\Lambda] A_{8}=-a^{6} \Upsilon^{\prime}[\Lambda] A_{2}+13 a^{2} \Upsilon^{\prime \prime}[\Lambda] A_{2}^{2}$ 


$$
\begin{aligned}
= & -a^{6} \Upsilon^{\prime}[\Lambda] A_{2}+\frac{A_{2}^{2}}{7}\left(7644 \Lambda \alpha_{3}-8280 \alpha_{2}\right. \\
& \left.+109 \Upsilon^{\prime \prime}[\Lambda]\right) a^{2} .
\end{aligned}
$$

If one wants $A_{2}$ to be arbitrary, since this might be later identified with the mass of the rotating black hole, the latter equation implies new constraints that relate the couplings of different orders in the curvature, which is incompatible with our previous assumption of genericity of the couplings. Consequently, we have proved that it is impossible to accommodate an asymptotically Kerr-AdS $\mathrm{A}_{5}$, rotating solution of cubic quasitopological theory within the Kerr-Schild Ansatz, for generic values of the couplings when the two rotation parameters are equal.

\section{CONCLUSIONS}

In this paper, we have constructed the slowly rotating solutions of cubic and quartic quasitopological gravities. The former is unique, while the latter is not. Namely, restricting first-order field equations on spherical symmetry singles out a unique cubic theory, which is also ghost free around AdS, while in the quartic theory both constraints lead to 13 restrictions on the 20 algebraically independent, Riem ${ }^{4}$ scalars in five dimensions. We have shown that the slowly rotating solution in the cubic theory is governed by a second-order equation, while in quartic quasitopological theories, requiring the equations to be of second order for the slowly rotating metric, implies one extra constraints on the couplings, leading to 14 conditions and therefore to a six-parameter family of Lagrangians. Even more, from the explicitly integration of the off-diagonal components, we have seen that three extra constraints could be imposed in order to achieve a simple off-diagonal metric functions, which allows an easy conjectural form for quasitopological gravities of arbitrary high order. In these computations, we have worked with the redundant list of 26 quartic scalars (the family $\mathcal{R}_{8,4}^{0}$ in the notation of [37]), since in this manner it will be now easy to find relations between Lagrangians of quartic theories in different dimensions. The same strategy allowed to prove that the dimensional reduction of the single cubic quasitopological theory in five dimensions [49] leads to the cubic combination with second-order equations on homogeneous and isotropic cosmologies in dimensions four identified and explored in [50-53]. It was also proved in [49] that the scalar gravitational perturbations on these FriedmannLemaître-Robertson-Walker (FLRW) background are governed by equations that are of second order in time. More recent explorations have shown that for vector and tensor modes this property might be absent in the cubic theory [54]. It would be interesting to explore whether these connections extend to the context of quartic theories and beyond. The freedom that still remains in the couplings can be used in one's favor at the moment of selecting gravitational theories with sensible properties. It is also interesting to mention that the classification of duality invariant $\alpha^{\prime}$ corrections in string inspired scenarios, in the cosmological Ansatz, has led to combinations of higher curvature terms with similar properties, namely, leading to second-order equations for the FLRW Ansatz [55]. Also, in the context of $\alpha^{\prime}$ corrections of string theory, it was proved in [56] that using the freedom of field redefinitions, intrinsic to the perturbative approach, every higher curvature combination can be rewritten in the frame of Einsteinian gravities, which also possess special properties on spherical symmetries [34,57-63]. In summary, there seem to be a network of theories with special properties in different dimensions, which deserves to be explored in its own right, and even more in the context of higher curvature, perturbative corrections to GR.

\section{ACKNOWLEDGMENTS}

We thank Pablo Bueno, José Edelstein, and Nicolás Grandi for enlightening comments. We specially thank Adolfo Cisterna for suggesting to analyze the equations of quasitopological gravities in the slowly rotating regime. This work was partially funded by FONDECYT Grants No. 1181047 and No. 11191175 . O. F. would like to thank the Direccion de Investigacion and Vicerrectora de Investigacion of the Universidad Catolica de la Santisima Concepcion, Chile for their constant support in particular through the Project DINREG 19/2018 of the Direccion de Investigacion of the Universidad Catolica de la Santisima Concepcion, Chile. N. M. thanks the support of Vicerrectoria de Investigacion, UdeC.

\section{APPENDIX A: QUARTIC CURVATURE SCALARS}

The following list defined the 26 invariants which are independent in dimension greater or equal than 8 . These correspond to the family $\mathcal{R}_{8,4}^{0}$ in the notation of [37] the following: 


$$
\begin{aligned}
& L_{1}=R^{p q b s} R_{p}{ }^{a}{ }^{u}{ }^{u} R_{a}{ }^{v} q^{w} R_{u v s w}, \quad L_{2}=R^{p q b s} R_{p}{ }^{a}{ }^{u} R_{a}{ }^{v}{ }^{w}{ }^{w} R_{q v s w}, \quad L_{3}=R^{p q b s} R_{p q}{ }^{a u} R_{b a}{ }^{v w} R_{\text {svuw }} \text {, } \\
& L_{4}=R^{p q b s} R_{p q}{ }^{a u} R_{b a}{ }^{v w} R_{\text {suvw }}, \quad L_{5}=R^{p q b s} R_{p q}{ }^{a u} R_{a u}{ }^{v w} R_{b s v w}, \quad L_{6}=R^{p q b s} R_{p q b}{ }^{a} R^{u v w}{ }_{s} R_{u v w a}, \\
& L_{7}=\left(R^{p q b s} R_{p q b s}\right)^{2}, \quad L_{8}=R^{p q} R^{b s a u} R_{b}{ }^{v}{ }_{a p} R_{\text {svuq }}, \quad L_{9}=R^{p q} R^{b s a u} R_{b s}{ }^{v}{ }_{p} R_{\text {auvq }}, \\
& L_{10}=R^{p q} R_{p}{ }^{b}{ }_{q}^{s} R^{\text {auv }}{ }_{b} R_{\text {auvs }}, \quad L_{11}=R R^{p q b s} R_{p}{ }^{a}{ }_{b}^{u} R_{\text {qasu }}, \quad L_{12}=R R^{p q b s} R_{p q}{ }^{a u} R_{b s a u} \text {, } \\
& L_{13}=R^{p q} R^{b s} R_{p}^{a}{ }_{b} R_{a q u s}, \quad L_{14}=R^{p q} R^{b s} R_{p}^{a}{ }_{p}^{u} R_{\text {abus }}, \quad L_{15}=R^{p q} R^{b s} R^{a u}{ }_{p b} R_{\text {auqs }}, \\
& L_{16}=R^{p q} R_{p}{ }^{b} R^{\text {sau }}{ }_{q} R_{\text {saub }}, \quad L_{17}=R^{p q} R_{p q} R^{\text {bsau }} R_{\text {bsau }}, \quad L_{18}=R R^{p q} R_{p}^{b s a} R_{b s a q}, \\
& L_{19}=R^{2} R^{p q b s} R_{p q b s}, \quad L_{20}=R^{p q} R^{b s} R_{b}{ }^{a} R_{p s q a}, \quad L_{21}=R R^{p q} R^{b s} R_{p b q s}, \\
& L_{22}=R^{p q} R_{p}{ }^{b} R_{q}{ }^{s} R_{b s}, \quad L_{23}=\left(R^{p q} R_{p q}\right)^{2}, \quad L_{24}=R R^{p q} R_{p}{ }^{b} R_{q b}, \quad L_{25}=R^{2} R^{p q} R_{p q}, \quad L_{26}=R^{4} .
\end{aligned}
$$

\section{APPENDIX B: COUPLINGS CONSTRAINTS}

On spherical symmetry, there are 11 independent constraints on the couplings of the quartic combinations that lead to a first-order equation for the function $f(r)$. The constraints are as follows:

$$
\begin{aligned}
& 0= 4 d_{4}+4 d_{6}+d_{20}+2 d_{15}+4 d_{18}+2 d_{23}+2 d_{10}+8 d_{19}+2 d_{16}+4 d_{9}+4 d_{25}+2 d_{2} \\
&+8 d_{5}+8 d_{7}+2 d_{21}+2 d_{13}+d_{14}+4 d_{17}+8 d_{26}+d_{22}+d_{1}+2 d_{24}+8 d_{12}, \\
& 0= 2 d_{10}+16 d_{12}+4 d_{13}+2 d_{14}+4 d_{15}+4 d_{16}+8 d_{17}+12 d_{18}+32 d_{19}+3 d_{20}+8 d_{21} \\
&+4 d_{22}+8 d_{23}+10 d_{24}+24 d_{25}+64 d_{26}+4 d_{9}, \\
& 0=4 d_{12}+2 d_{18}+8 d_{19}+d_{21}+d_{24}+4 d_{25}+16 d_{26}, \\
& 0=4 d_{2}+28 d_{18}+54 d_{24}+38 d_{21}+2 d_{3}+32 d_{7}+576 d_{26}+112 d_{19}+7 d_{13}+9 d_{14}+4 d_{1}+8 d_{6} \\
&+ 164 d_{25}+2 d_{8}+6 d_{11}+18 d_{22}+28 d_{17}+44 d_{23}+8 d_{16}+11 d_{20}+6 d_{10}+6 d_{15}, \\
& 0= 2 d_{10}+2 d_{14}+8 d_{17}+6 d_{18}+48 d_{19}+d_{20}+10 d_{21}+8 d_{23}+9 d_{24}+56 d_{25}+288 d_{26}, \\
& 0=3 d_{17}+8 d_{19}+2 d_{23}+5 d_{25}+36 d_{26}+4 d_{7}, \\
& 0= 25 d_{1}+4 d_{10}-36 d_{11}+47 d_{13}+32 d_{15}+62 d_{16}-148 d_{17}+66 d_{18}-768 d_{19}+30 d_{2} \\
&+30 d_{20}-78 d_{21}+135 d_{22}-82 d_{23}+123 d_{24}-916 d_{25}-7488 d_{26}+8 d_{3}+68 d_{6}, \\
& 0= d_{1}+12 d_{11}+11 d_{13}+8 d_{15}+14 d_{16}+12 d_{17}+62 d_{18}+288 d_{19}-2 d_{2}+12 d_{20}+54 d_{21} \\
&+ 39 d_{22}+30 d_{23}+117 d_{24}+300 d_{25}+1152 d_{26}+4 d_{6}, \\
& 0= 6 d_{1}-14 d_{10}+24 d_{13}+12 d_{15}+36 d_{16}-76 d_{17}+22 d_{18}-384 d_{19}+12 d_{2}+21 d_{20} \\
&+6 d_{21}+102 d_{22}+44 d_{23}+153 d_{24}-4 d_{25}-960 d_{26}+24 d_{6}, \\
& 0= 14 d_{10}+18 d_{13}-12 d_{15}+48 d_{16}+148 d_{17}+134 d_{18}+768 d_{19}-24 d_{2}+15 d_{20}+30 d_{21} \\
&+ 180 d_{22}+136 d_{23}+393 d_{24}+556 d_{25}+576 d_{26},
\end{aligned}
$$

From (22), there are two new constraints such that the linearized equations around AdS are of second order. These are follows: 


$$
\begin{aligned}
0= & -8 d_{13}-6 d_{15}-10 d_{16}-22 d_{17}-46 d_{18}-248 d_{19}-7 d_{20}-16 d_{21}-28 d_{22}-16 d_{23} \\
& -71 d_{24}-74 d_{25}+272 d_{26}, \\
0= & -4 d_{13}-4 d_{15}-4 d_{16}-8 d_{17}-22 d_{18}-112 d_{19}-5 d_{20}-14 d_{21}-12 d_{22}-12 d_{23} \\
& -39 d_{24}-72 d_{25}+96 d_{26} .
\end{aligned}
$$

The new constraint, that leads to a second-order equation for the off-diagonal metric component of the slowly rotating metric $h_{i}$, is

$$
0=d_{20}+4 d_{22}-4 d_{23}+3 d_{24}-22 d_{25}-144 d_{26}+4 d_{6}+3 d_{16}-10 d_{17}-2 d_{18}-72 d_{19}
$$

[1] D. Lovelock, J. Math. Phys. (N.Y.) 12, 498 (1971).

[2] B. Zumino, Phys. Rep. 137, 109 (1986).

[3] C. Garraffo and G. Giribet, Mod. Phys. Lett. A 23, 1801 (2008).

[4] C. Charmousis, Higher order gravity theories and their black hole solutions, in Physics of Black Holes, Lecture Notes in Physics Vol. 769 (Springer, Berlin, Heidelberg, 2009), pp. 299-346.

[5] M. Brigante, H. Liu, R. C. Myers, S. Shenker, and S. Yaida, Phys. Rev. D 77, 126006 (2008).

[6] R. R. Metsaev and A. A. Tseytlin, Nucl. Phys. B293, 385 (1987).

[7] X. O. Camanho, J. D. Edelstein, J. Maldacena, and A. Zhiboedov, J. High Energy Phys. 02 (2016) 020.

[8] S. Willison, Classical Quant. Grav. 32, 022001 (2015).

[9] G. Papallo and H. S. Reall, Phys. Rev. D 96, 044019 (2017).

[10] T. Andrade, E. Caceres, and C. Keeler, Classical Quant. Grav. 34, 135003 (2017).

[11] R. R. Metsaev and A. A. Tseytlin, Phys. Lett. B 185, 52 (1987).

[12] J. Oliva and S. Ray, Classical Quant. Grav. 27, 225002 (2010).

[13] R. C. Myers and B. Robinson, J. High Energy Phys. 08 (2010) 067.

[14] Y. Brihaye and E. Radu, Phys. Lett. B 661, 167 (2008).

[15] R. A. Konoplya and A. Zhidenko, Phys. Rev. D 102, 084030 (2020).

[16] H. C. Kim and R.G. Cai, Phys. Rev. D 77, 024045 (2008).

[17] R. Yue, D. Zou, T. Yu, P. Li, and Z. Yang, Gen. Relativ. Gravit. 43, 2103 (2011).

[18] A. Anabalon, N. Deruelle, Y. Morisawa, J. Oliva, M. Sasaki, D. Tempo, and R. Troncoso, Classical Quant. Grav. 26, 065002 (2009).

[19] M. Cvetic, X. H. Feng, H. Lu, and C. N. Pope, Phys. Lett. B 765, 181 (2017).

[20] A. Anabalon, N. Deruelle, D. Tempo, and R. Troncoso, Int. J. Mod. Phys. D 20, 639 (2011).
[21] M. H. Dehghani, A. Bazrafshan, R. B. Mann, M. R. Mehdizadeh, M. Ghanaatian, and M. H. Vahidinia, Phys. Rev. D 85, 104009 (2012).

[22] A. Cisterna, L. Guajardo, M. Hassaine, and J. Oliva, J. High Energy Phys. 04 (2017) 066.

[23] P. Bueno, P. A. Cano, and R. A. Hennigar, Classical Quant. Grav. 37, 015002 (2020).

[24] M. Chernicoff, O. Fierro, G. Giribet, and J. Oliva, J. High Energy Phys. 02 (2017) 010.

[25] H. Dykaar, R. A. Hennigar, and R. B. Mann, J. High Energy Phys. 05 (2017) 045.

[26] M. Hassaine and J. Zanelli, Chern-Simons (Super)Gravity (World Scientific, Singapore, 2016).

[27] J. T. Wheeler, Nucl. Phys. B268, 737 (1986).

[28] J. T. Wheeler, Nucl. Phys. B273, 732 (1986).

[29] H. Maeda, S. Willison, and S. Ray, Classical Quant. Grav. 28, 165005 (2011).

[30] X. O. Camanho and J. D. Edelstein, J. High Energy Phys. 06 (2010) 099.

[31] X. O. Camanho and J. D. Edelstein, Classical Quant. Grav. 30, 035009 (2013).

[32] C. Corral, D. Flores-Alfonso, and H. Quevedo, Phys. Rev. D 100, 064051 (2019).

[33] D. Klemm, V. Moretti, and L. Vanzo, Phys. Rev. D 57, 6127 (1998); 60, 109902(E) (1999).

[34] C. Adair, P. Bueno, P. A. Cano, R. A. Hennigar, and R. B. Mann, Phys. Rev. D 102, 084001 (2020).

[35] R. S. Palais, Commun. Math. Phys. 69, 19 (1979).

[36] S. Deser and J. Franklin, Am. J. Phys. 73, 261 (2005).

[37] S. A. Fulling, R. C. King, B. G. Wybourne, and C. J. Cummins, Classical Quant. Grav. 9, 1151 (1992).

[38] P. Bueno, P. A. Cano, V. S. Min, and M. R. Visser, Phys. Rev. D 95, 044010 (2017).

[39] G. W. Gibbons, H. Lu, D. N. Page, and C. N. Pope, Phys. Rev. Lett. 93, 171102 (2004).

[40] G. W. Gibbons, H. Lu, D. N. Page, and C. N. Pope, J. Geom. Phys. 53, 49 (2005).

[41] E. Ayón-Beato, M. Hassaïne, and D. Higuita-Borja, Phys. Rev. D 94, 064073 (2016). 
[42] B. Carter, Commun. Math. Phys. 10, 280 (1968).

[43] R. C. Myers and M. J. Perry, Ann. Phys. (N.Y.) 172, 304 (1986).

[44] S. W. Hawking, C. J. Hunter, and M. Taylor, Phys. Rev. D 59, 064005 (1999).

[45] B. Ett and D. Kastor, J. High Energy Phys. 04 (2011) 109.

[46] E. Babichev, C. Charmousis, A. Cisterna, and M. Hassaine, J. Cosmol. Astropart. Phys. 06 (2020) 049.

[47] A. Cisterna, M. Cruz, T. Delsate, and J. Saavedra, Phys. Rev. D 92, 104018 (2015).

[48] J. Crisostomo, R. Troncoso, and J. Zanelli, Phys. Rev. D 62 , 084013 (2000).

[49] A. Cisterna, N. Grandi, and J. Oliva, Phys. Lett. B 805, 135435 (2020).

[50] G. Arciniega, J. D. Edelstein, and L. G. Jaime, Phys. Lett. B 802, 135272 (2020).

[51] G. Arciniega, P. Bueno, P. A. Cano, J. D. Edelstein, R. A. Hennigar, and L. G. Jaime, Phys. Lett. B 802, 135242 (2020).

[52] G. Arciniega, P. Bueno, P. A. Cano, J. D. Edelstein, R. A. Hennigar, and L. G. Jaime, Int. J. Mod. Phys. D 28, 1944008 (2019).
[53] J. D. Edelstein, D. Vázquez Rodríguez, and A. Vilar López, J. Cosmol. Astropart. Phys. 12 (2020) 040.

[54] M. C. Pookkillath, A. De Felice, and A. A. Starobinsky, J. Cosmol. Astropart. Phys. 07 (2020) 041.

[55] O. Hohm and B. Zwiebach, Phys. Rev. D 100, 126011 (2019).

[56] P. Bueno, P. A. Cano, J. Moreno, and Á. Murcia, J. High Energy Phys. 11 (2019) 062.

[57] P. Bueno and P. A. Cano, Phys. Rev. D 94, 104005 (2016).

[58] R. A. Hennigar and R. B. Mann, Phys. Rev. D 95, 064055 (2017).

[59] P. Bueno and P. A. Cano, Phys. Rev. D 94, 124051 (2016).

[60] P. Bueno and P. A. Cano, Classical Quant. Grav. 34, 175008 (2017).

[61] P. Bueno, P. A. Cano, and A. Ruipérez, J. High Energy Phys. 03 (2018) 150.

[62] P. A. Cano and D. Pereñiguez, Phys. Rev. D 101, 044016 (2020).

[63] R. A. Hennigar, D. Kubiž̌ák, and R. B. Mann, Phys. Rev. D 95, 104042 (2017). 